\section{EFFECT OF 'TRAINING'}

Fig. 5 shows a qualitative description of the influence of fitness on the heart movements changes during exercise. Fig. 5a is a measurement taken from a person who is completely unfit and a heavy smoker. One can note the extreme values of the ' $a$ ' wave after 60 minutes of work. Fig. 5b shows the heart movements recorded over the same subject after 7 consecutive days of exercise. Note that the ' $a$ ' wave has significantly reduced its amplitude. Fig. 5c shows the heart movement obtained from a very fit person in the first day and after 60 minutes of exercise.

\section{DISCUSSION}

Presented here is a method of estimating the heart load during exercise of normal subjects. The changes of the ' $a$ ' wave during exercise as can be seen from Fig. 4 are much higher than those of the heart rate and whereas heart rate tends to equilibriate after some time of exercise, the changes in the ' $a$ ' wave keep on rising. This suggests that the measurement of the 'a' wave during exercise could become a better means of assessing the condition of certain hearts during physical exercise than does the heart rate. However, the results described here are preliminary and much more work should be put into this subject to reach eventually a reliable index of 'cardiac fitness'.

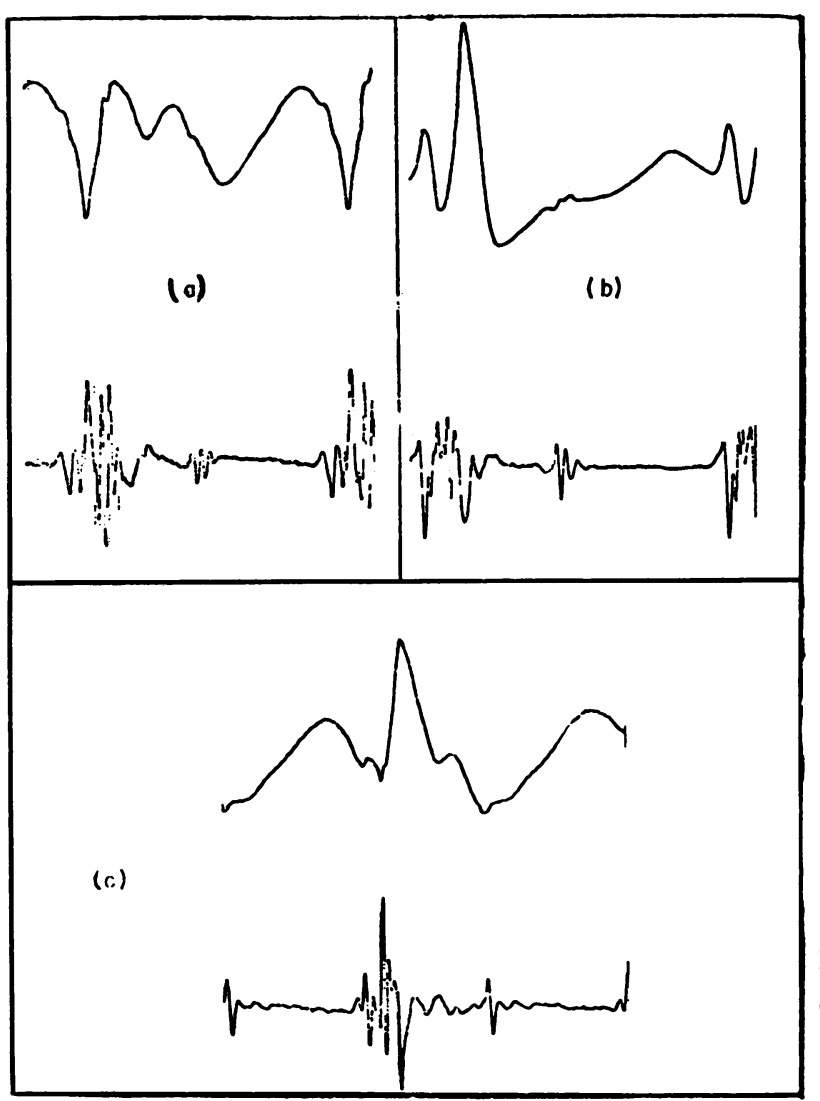

Fig. 5

\title{
THE USE AND ABUSE OF STATISTICAL METHODS IN RESEARCH
}

\author{
M. M. JORDAN, M.Sc.
}

Statistical methods must always be used with care and with full knowledge of their limitations. The use of these methods without consideration of the necessary conditions which must exist for them to be applicable has led to a general scepticism of statistical techniques. This is regrettable as such techniques are a most useful research tool.

I propose to outline in this paper the more common fallacies which arise in the application of statistical methods and to show that it is the misapplication of such methods and not the methods themselves which are worthy of scepticism.

\section{PRESENTATION OF DATA}

Statistical results are often presented in pictorial form, e.g. as pie charts, histograms, or graphs of relationships between variables, to make the information more digestible to the reader. For example, the pie chart (Fig. 1) is a very simple method of displaying data which might otherwise be a confusing mass of figures. The histogram or bar chart (Fig. 2) is another method of presenting the same sort of data but it is important to remember that it is the areas of the rectangles which represent the relative frequencies and, therefore, the widths of the rectangles should be kept constant. In 


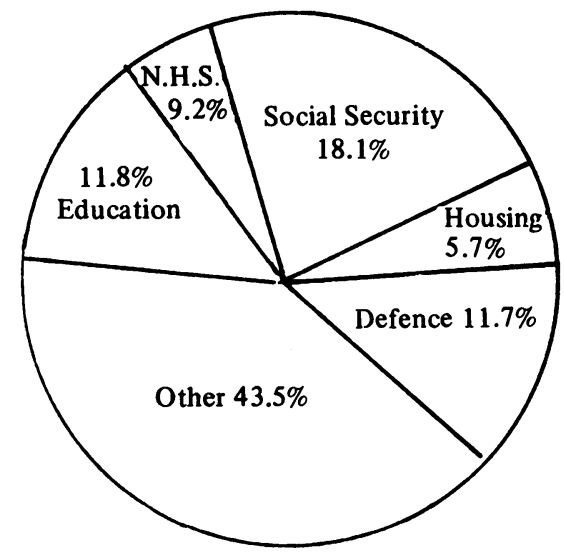

FIG. 1 Pie Chart

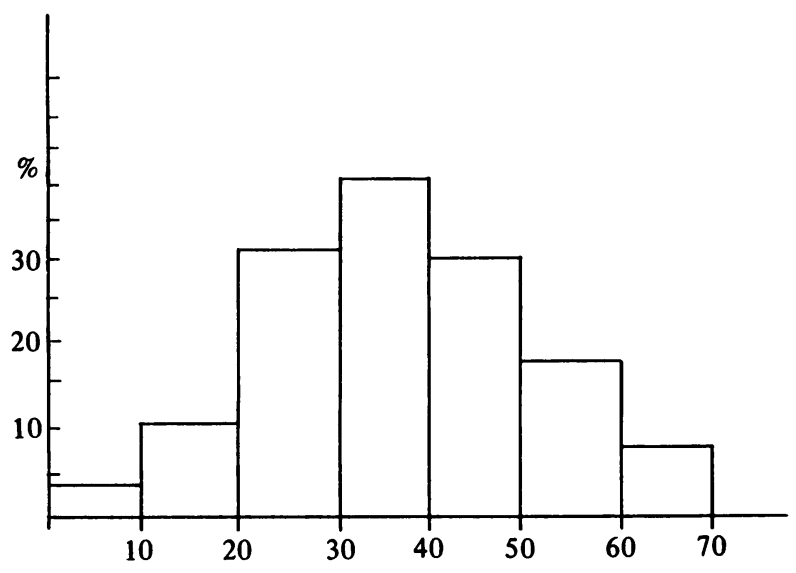

FIG. 2 - Histogram or Bar Chart

addition, if two such charts with unequal numbers of cases are compared, the frequencies should be expressed as percentages and not as absolute frequencies before the comparison is made.

The researcher tends to use graphs more than the previous two methods, sometimes with surprising results. In efforts to show relationships, graphs are presented either with no scale or with unequal scales (compare Figures $3 a$ and $3 b$ ). There is a great psychological urge to draw a "smooth curve" through a series of points which often show no more trend than snowflakes in a storm. Moreover, it is not unknown for an enthusiastic researcher to ignore a few awkward points which do not fit the pattern he expects. Similar to this is the tendency to extrapolate, i.e. to extend the graph beyond the range of factual information. For example, Fig. 4 shows the years of expectation of life in males from 1901 to 1966.
If in 1951, the current trend had been extrapolated, the result would have been as shown by the dotted line which produces a much higher value in 1966 than was actually found. This example stresses the fact that all extrapolation should be carefully distinguished on a graph, e.g. using a dotted line as contrast to a full line in the rest of the graph.

\section{GROWTH RATE}

(a)

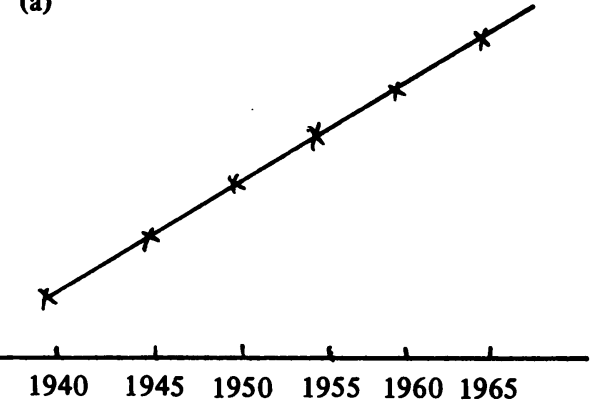

GROWTH RATE

(b)

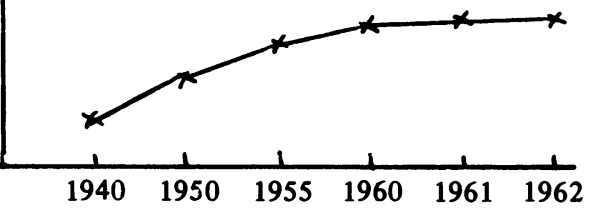

FIG. 3 - Effect of a Scale Change

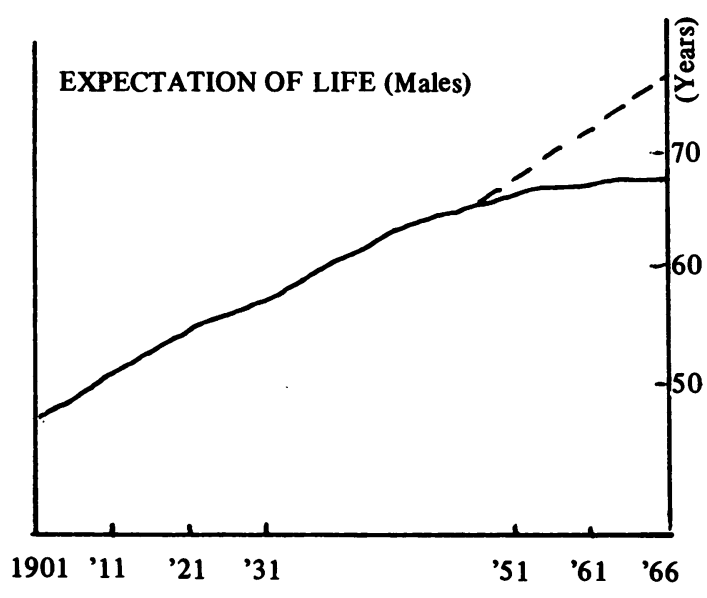

FIG. 4 - Extrapolation 


\section{CONCEPT OF AVERAGE AND VARIANCE}

The researcher often needs to reduce his data to a few parameters which allow him to examine it more clearly. One such concept is the "average" or "measure of central tendency". There are several types of averages, the most common being the arithmetic mean. The example that there are 2.2 children in the average family demonstrates the rather abstract quality of this concept and the care with which it should be treated.

A common mistake when dealing with averages is to consider the sum of averages of a population as the average of the whole population. For example, an aeroplane covers 300 miles in three stages:

(a) First 100 miles at 100 m.p.h.

(b) Second 100 miles at 200 m.p.h.

(c) Third 100 miles at 300 m.p.h.

The average speed over the whole journey is often calculated

$$
\frac{100+200+300}{3}=200 \text { m.p.h. }
$$

However, if we consider the problem more clearly as follows:

(a) Time to travel $1 \mathrm{st} 100$ miles $=1$ hour

(b) Time to travel 2 nd 100 miles $=30$ minutes

(c) Time to travel 3 rd 100 miles $=20$ minutes Total time

$=1$ hour 50 minutes $=11 / 6$ hours

Average velocity $=\frac{300}{11 / 6}$ $=164$ m.p.h.

Table 1 shows a similar example. In this table, the total expenditure of an average family per week is calculated as the sum of the average amounts spent on various commodities. In addition to the sum of averages being wrong, the figures themselves are open to criticism. They came from many different and possibly biased sources and there is no attempt to show the range of expenditure on any one item.

\section{TABLE 1 - Expenditure Per Week in Average Family}

Family Car (R.A.C.)

Food

Council House Rents (Government)

Travel Agents

Clothing (Manufacturers)

Cigarettes

Drink

Pet Food (Manufacturers)

Children's Pocket Money (Newspaper Survey)

School Meals

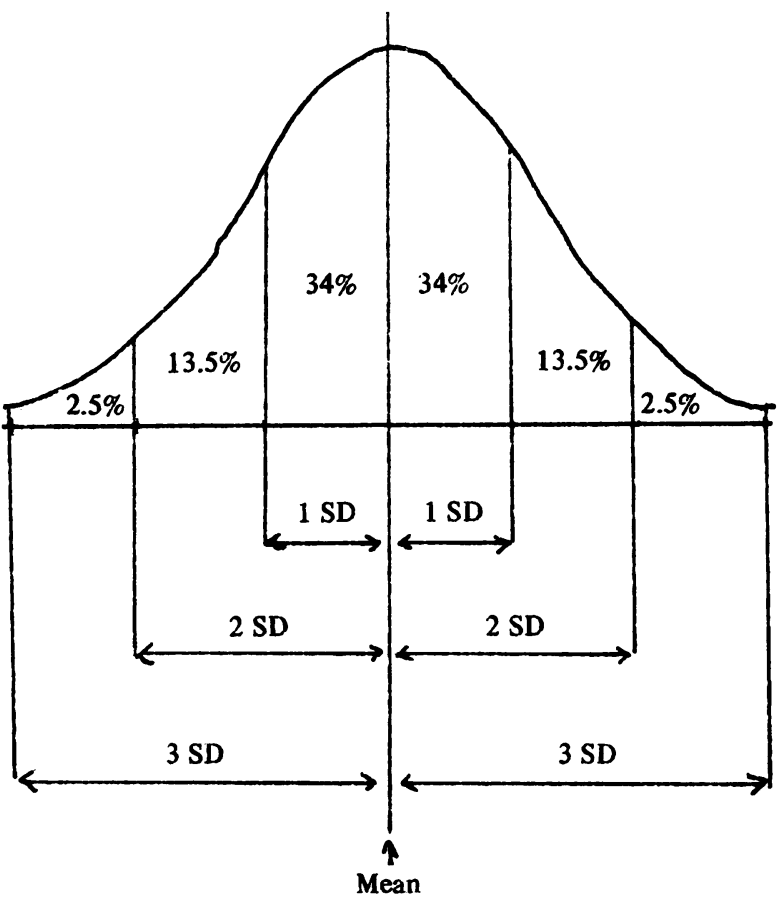

FIG. 5 - Use of Standard Deviation in defining a Distribution

This brings us to the concept of variance. Associated with an average, the researcher must have some estimate of the scatter of values about this central value. For example, to say that a group of men had an average age of 45 tells one nothing about the age range of the group which could be from 44 to 46 or 10 to 70 . Again, several different estimates of variance are used. They can be the range itself, the mean deviation, * or more often the standard deviation.** This last parameter is an extremely useful measure of variance but care must be taken in interpreting the results if the distribution of the population is not normal. If the distribution is normal, the standard deviation can be used to define the scatter of the population as illustrated in Fig. 5 .

\section{CONCEPT OF CORRELATION}

Another important concept in research is that of correlation usually calculated using the correlation coefficient? It is worthwhile noting that this coefficient only measures a linear relationship between two variables and will not establish a non-linear relationship.

The whole concept of relationships between variables is very important in research and accordingly great care should be taken in accepting such relationships. Often, a correlation between two events is not a result of a relationship between them but is due to a third event which affects both the other two. For example, it is possible to show a strong correlation between the 
increase in teachers' salaries over the last 20 years and the increase in the consumption of alcohol. The latter increase is due, we hope to the general rise in the standard of living during this time and not to the teaching community in particular.

This example shows the need to consider all influencing factors in a research investigation for of ten the common factor causing apparently significant correlations is not obvious. It has been clear for some time, for example, that heavy smokers tended to have lung cancer more often than non-smokers but it has taken more than 20 years of research to prove that this relationship was not due to a chance effect of atmospheric pollution, occupational hazards, genetic tendencies, etc.

\section{SURVEY TECHNIQUE - SAMPLING}

A statistical technique available to all researchers at little cost is that of surveys. Armed with a paper and pencil, anyone can conduct his own piece of research whether it be on the increase in food prices over six months or the effect of pollution on our environment. This technique, however, is full of dangers to the inexperienced and it is of ten wise to consult a qualified statistician before the investigation is underway. This consultation can be particularly helpful in establishing the sampling technique to be used. Sampling method is completely dependent on the investigation and it is impossible to classify all the variations used by researchers at present. I have attempted to summarise the more general types and these are as follows:

1. Universal - where the whole population is considered, e.g. in a census. This is used in research where the incidence of the condition of interest is very rare in the population.

2. Completely random - where individuals are selected using a random selection device, e.g. a table of random numbers. For example, the houses in a street may be selected by using a table of random numbers to select the house numbers.

3. Stratified - groups are defined and individuals selected at random from each group. For example, an investigator may divide his population into age groups and select randomly from each group.

4. Sequential - taking sequential samples at fixed intervals. For example, the extended census form is given to $10 \%$ of the population. Each enumerator is given a bundle of forms of which every tenth one is a $10 \%$ census form. He is then asked to remove $\mathrm{x}$ forms from the top of the bundle, where $x$ is a random number less than 10 , leaving the extended census forms in the (10-x)th, $(20-x)$ th, etc. positions in the bundle. He is then asked to visit each household as instructed supplying each with the form from the top of the bundle.

Each type of sampling is appropriate under different circumstances and it is only with experience that the researcher can decide how to sample his population. When the sampling process is complete, the sample should always be compared with the whole population as far as it is possible to ensure that a representative sample has been obtained. The parameters often used for this comparison are age, sex, social class, etc. The example in Table 2 illustrates the need for these checks. The table shows the results of a test of a measles vaccine in which the vaccinated population were chosen at random. The table shows that those children vaccinated had a higher incidence of the disease than those not vaccinated. These results were very puzzling until the investigators looked at the number of children in the family in the two groups with the hypothesis that one child families should not be subject to the same risk of infection. The tables shows that there was, as suspected, a much higher incidence of one child families in the uninnoculated group. When the figures are broken down by size of family, no difference between the groups is demonstrable.

\section{TABLE 2 - Results of Measles Vaccine Trial}

INOCULATED

$\begin{array}{lll}\text { Attacked } & 96(48 \%) & 72(36 \%) \\ \text { Not Attacked } & 104(52 \%) & 128(64 \%) \\ \text { TOTAL } & 200 & 200\end{array}$

No. of Children

Children in
Family Attacked
1
2
3
$4+$

TOTAL in Family

\section{$\underset{\%}{\text { INOCULATED }} \underset{\%}{\text { NOT INOCULATED }}$ \\ $\%$}

18

46

22

14

100

100

50

20

16

14
TOTAL

INOCULATED NOT INOCULATED
16 (16\%)

$20(50 \%)$

$16(50 \%)$

20 (71\%)

72 


\section{OTHER TECHNIQUES}

In addition to the simple statistical methods used in research, there are techniques which allow the researcher to examine many variables simultaneously and so obtain an overall picture of the relationship between them. Although these techniques are powerful and are a useful tool to the researcher, they will never replace simple, careful examination of data.

Finally, a word of advice to those who are developing an interest in using statistical methods in their research. The consultation of an experienced statistician in the initial stages of your investigation can help you avoid the difficulties which the researcher meets in work of this type. The experienced statistician can not only explain the techniques you should use but will also point out any assumptions which are needed for these methods. In this way, you ensure that your results are clearly displayed and, more important, statistically valid.

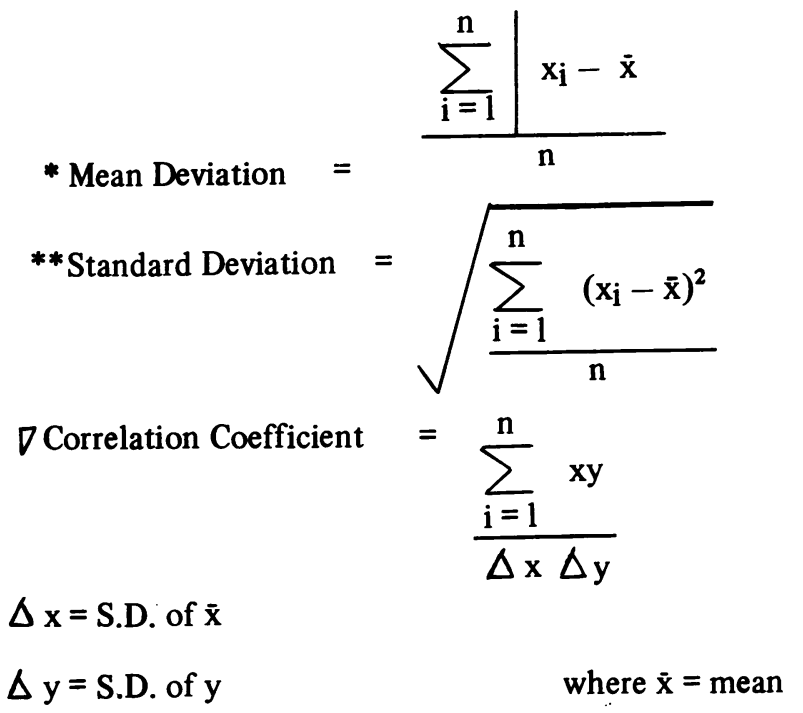

\section{ANNOTATION \\ A.I.B.A. MEDICAL COMMISSION \\ Report on injuries received during the Olympic Games, Munich, 1972. \\ J. L. BLONSTEIN, Medical Advisor to A.B.A. and President A.I.B.A. Medical Commission}

There were 357 boxers and 347 bouts.

There were 4 boxers with haematomata (blood swellings), 1 bout being stopped.

There were 13 cuts in the region of the eye for which the bouts were stopped.

There were 4 boxers with abrasions, none being stopped.

3 boxers retired, 1 with a cut left eye, 1 unable to go on and 1 with an injury to his left arm.

There were 21 boxers with small cuts which were not stopped.

There were 21 knock-outs, 10 in the first round, 8 in the second round and 3 in the third round.

2 boxers were unconscious for 2 minutes but recovered rapidly in the ring. 1 was K.O.'d by a solar plexus punch.

2 boxers were sent to hospital but neither was detained. 1 became hysterical after his bout and the other had sustained a blow to the liver.

There was 1 partial dislocation of the left shoulder, 1 Bennet's fracture of the right thumb, 1 fractured metacarpal of the right hand, 1 sprained ankle and 1 fractured nose. 1 boxer lost 2 teeth during his bout.

99 boxers were given anti-doping tests but none were positive. 